\title{
Post-LASIK IOL Power Calculations: Where Are We in 2012
}

\author{
Maya C. Shammas $\cdot$ H. John Shammas
}

Published online: 17 January 2013

(c) Springer Science + Business Media New York 2013

\begin{abstract}
Calculating the correct intraocular lens (IOL) after refractive surgery, whether it is PRK, LASIK, LASEK, or RK, can be a challenge. This review will aim to give an understanding of the errors and how it affects the formulas used in IOL power calculations. This will aid ophthalmologists in choosing the best IOL for their patients.
\end{abstract}

Keywords IOL power calculations - Prior refractive surgery · Myopic LASIK · Hyperopic LASIK .

Myopic PRK · Hyperopic PRK · RK

\section{Introduction}

Calculating the correct intraocular lens (IOL) for cataract surgery has become even more crucial for a successful practice as patients more and more desire emmetropia or a certain refractive outcome for at least distance. Patients who have had corneal refractive surgery have already experienced emmetropia, and they will expect similar results after cataract surgery. Unfortunately, these are the hardest cases to predict the best IOL.

Refractive surgery comprises mostly LASIK, LASEK, and PRK. Studies have shown that between these types of refractive surgery, there is no significant difference in the degree of errors [1•]. We will therefore look at these as essentially one entity, termed LASIK/PRK. There does tend

M. C. Shammas $(\bowtie)$

Shammas Medical Eye Center, 3510 Martin Luther

King Junior Boulevard, Lynwood, CA 90262, USA

e-mail: mayashammas@gmail.com

H. J. Shammas

The Keck School of Medicine of USC, 3510 Martin Luther

King Junior Boulevard, Lynwood, CA 90262, USA to be a difference when looking at a patient post-myopic laser vision correction or post-hyperopic laser vision correction. Also, radial keratotomy, RK, causes a different affect on the cornea and results in a different type of error. While RK is no longer in favor, we still encounter these patients and need to know the best way to calculate the IOL in all these cases.

In post-myopic LASIK/PRK patients, there are essentially two errors that are produced. The first error comes from the measurement of the corneal power or $\mathrm{K}$ readings, while the second error comes from the measurement of the estimated lens position, ELP [2-7]. These two errors, the K readings and ELP miscalculation, are additive. The intraocular lens is underestimated leading to a post-operative hyperopic surprise.

Many formulas have been developed in order to correct these errors. In addition, advancements in technology have aided in giving us more information and more precise measurements. In order to make the best choice in IOL for your patient, understanding all the data collected and how to utilize them will help in making an informed decision. In this review, an understanding will be given of how the data are obtained, where the errors in the calculations originate from, and how best to correct them.

\section{Obtaining the Pre-Operative Data}

In all patients undergoing cataract surgery, routine measurements include the axial length, the anterior chamber depth, and the corneal power.

Measuring the Axial Length

Multiple options are available when it comes to measuring the axial length and the anterior chamber depth, ACD [8]. 
Ultrasound biometry has been used longer than any other method. An ultrasound beam is emitted from the transducer at the tip of the probe, and an A-Scan pattern is displayed on the screen. The measurements are taken in microseconds and converted to millimeters using a velocity of $1,532 \mathrm{~m} / \mathrm{s}$ for the aqueous and vitreous and $1,641 \mathrm{~m} / \mathrm{s}$ for the cataractous lens. Of the different types of ultrasound biometry, immersion ultrasonography is the preferred method, whereas contact biometry can be less accurate especially in myopic cases with a possible staphyloma.

Optical biometry, such as the IOLMaster (Carl Zeiss Meditec), has replaced ultrasound as the standard technique to measure the axial length. It uses partial coherence interferometry with a $780-\mu \mathrm{m}$ laser diode infrared light to measure the axial length. A lateral slit beam of light is used to measure the anterior chamber depth. The anterior chamber depth is defined as the measurement between the corneal epithelium and the anterior lens surface. This technology relies on the ability of light to penetrate the cataractous lens, and thus is not able to take measurements in very mature cataracts.

The most recent technology to become available is the Lenstar LS 900 optical biometer (Haag-Streit). This technology utilizes an $820-\mu \mathrm{m}$ super luminescent diode and takes multiple measurements in a single step. The principle is based on optical low-coherence reflectometry, OLCR. It measures the axial length, the central corneal thickness, the lens thickness, and aqueous depth. Of note, the aqueous depth using this apparatus is defined as the measurement from the corneal endothelium to the anterior lens surface. The anterior chamber depth is displayed by adding the corneal thickness to the aqueous depth.

\section{Measuring the Corneal Power}

The keratometer is an instrument that measures the anterior radius of corneal curvature, expressed in millimeters. This number is translated into diopters by using an equation that considers the entire corneal power to be at the anterior corneal surface and a 1.3375 index of refraction. Different types of keratometry exist including manual and automated keratometry.

Manual keratometry is the oldest method used to measure the corneal curvature, and many formulas have been based on the measurements from manual keratometry. Four points on two orthogonal meridians are measured, separated by 3-4 $\mathrm{mm}$ on the paracentral cornea. These measurements do not seem to be more accurate than the automated counterpart and may have more variability in the readings [9]. The one advantage is that one can see the mires and irregularity can be visualized [10].

Automated keratometry has gained in popularity in the past two decades. Most auto-keratometers are either stand- alone units or combined with an auto-refractor. They provide the $\mathrm{K}$ readings from the central $2.6-3 \mathrm{~mm}$ of the cornea in the steepest and flattest meridians. The IOLMaster added an auto-keratometer to its optical biometer, making it more convenient to take all required measurements from one instrument. The $\mathrm{K}$ readings are calculated by analyzing the anterior corneal curvature at 6 reference points oriented in a hexagonal pattern at approximately the 2.3-mm optical zone. With the Lenstar, the $\mathrm{K}$ readings are calculated by analyzing the anterior corneal curvature at 32 reference points orientated in two circles at approximately the 2.30 - and $1.65-\mathrm{mm}$ optical zones. Post-refractive surgery, errors can occur due to instrument error specifically when measurements are taken at the margin of the treatment zone. Because automated keratometers take measurements from a smaller optical zone as compared to manual keratometry, these measurements tend to be better post-refractive surgery [9].

Corneal topography is another tool that gives additional information that can be extremely valuable in the postrefractive patient. Measurements of corneal power, simulated keratometry, or Sim-K, are taken from the placido mires of the videokeratoscope and sample more than 1,000 points over the central cornea [8]. This may include the central 1,2 , and $3 \mathrm{~mm}$ depending on the instrument being used. It also gives a more accurate evaluation and map of any pre-existing astigmatism.

Scheimpflug photography is used in the Pentacam device (Oculus) and produces a 3-dimensional analysis of the anterior segment. The anterior corneal surface is measured producing a simulated keratometry value, or sim-K, which is what has been used for IOL power calculation. Other measurements taken include the posterior corneal radius, anterior corneal radius, and central corneal thickness. The program has used these measurements to calculate an equivalent $\mathrm{K}$ reading labeled the Holladay Report. The equivalent $\mathrm{K}$ readings supposedly reflect the correct $\mathrm{K}$ values in post-refractive cataractous eyes. However, there are no definite studies establishing its validity. The computer software also calculates the total corneal power called the true net power. This measurement is not recommended for use in routine intraocular lens power calculations because all commonly used IOL power formulas require the corneal vertex power based on a 1.3375 index of refraction, not the total corneal power.

\section{Post-Myopic Refractive Surgery}

\section{Corneal Changes After Myopic Refractive Surgery}

Myopic refractive surgery flattens the anterior cornea in order to correct the existing refractive error. The posterior 
cornea is left unchanged, altering the normal anterior to posterior corneal curvature ratio $[11,12]$. The shape of the cornea has changed from sphere-cylindrical to irregular $[2,13]$. These changes cause an overestimation of the measured corneal power after myopic LASIK and PRK.

The air to anterior corneal interface provides approximately 49 diopters of power while the posterior corneal to aqueous interface takes away about six diopters. Traditional keratometers measure the anterior cornea surface only and utilize the normal relationship between the anterior and posterior surface to correct the difference. The average ratio of anterior to posterior curvature is 1.21 . After myopic laser refractive surgery, this number increases proportional to the amount of corneal flattening.

The radius of corneal curvature, $r$, is taken in millimeters and then translated into diopters of corneal power, $K$, using an index of refraction of 1.3375 . The relationship is

$K=1,000(1.3375-1) / r$

After myopic PRK and LASIK, the corneal changes mentioned above, in addition to decreasing the distance between the anterior and posterior surface, decrease the effective corneal index of refraction. The overall result is higher measured corneal power than actually exists. A classical example would be the cornea with a pre-LASIK K of $45.00 \mathrm{D}$. After a 5.00-D myopic correction at the corneal level, one would expect the post-LASIK K to be $40.00 \mathrm{D}$. Instead, the measured $K$ is $41.25 \mathrm{D}$, an overestimation of $1.25 \mathrm{D}$. Studies have shown that the $K$ readings need to be decreased by a factor of $0.23 \mathrm{D}$ for each diopter of myopic correction obtained by the refractive surgery. Scheimpflug photography can now accurately measure not only the anterior corneal radius but also the posterior one to obtain an exact measurement of the total corneal power.

\section{Correcting the Errors}

If knowledge of the pre-LASIK keratometry values, $K$, and the change in manifest refraction, MR, is known, one can calculate the IOL power utilizing the clinical history method to correct the corneal power and Aramberri's double-K method to correct the ELP. The double-K method is only needed if using the SRK/T, Holladay 1 , or Hoffer Q formulas and is not needed when using the Haigis or Shammas-PL formula. This is because these formulas do not depend on the corneal curvature to calculate ELP.

\section{Clinical History Method to Correct the Corneal Power}

The clinical history method is an accurate way of correcting the overestimation of the corneal power after refractive surgery. The corrected corneal power, Kc, is obtained using the pre-LASIK $\mathrm{K}$ and the change in refractive error at the corneal plane, CRc.

The first step is to determine the change in refractive error at the corneal plane. Convert the pre-LASIK and postLASIK manifest refraction at the spectacle plane, Rs, to the manifest refraction at the corneal plane, Rc. To do this, one can assume a vertex distance of $12 \mathrm{~mm}$ and use the equation [14]:

$\mathrm{Rc}=\mathrm{Rs} /(1-0.12 \mathrm{Rs})$

Then, take the difference between the pre-LASIK and post-LASIK manifest refraction to determine the change in refraction at the corneal plane, CRc.

$\mathrm{CRc}=\mathrm{Rc} \cdot$ post - Rc.pre

Now, the corrected corneal power, Kc, can be calculated by algebraically adding the difference in refractive error to the pre-LASIK corneal power, Kpre. Of note, with myopic correction, the change in refraction is a negative number, so the amount will essentially be subtracted from the preLASIK corneal power, whereas with hyperopic correction, there is a positive change in refraction and the amount will be added to the pre-LASIK corneal power.

$\mathrm{Kc}=\mathrm{Kpre}-\mathrm{CRc}$ (after myopic correction)

$\mathrm{Kc}=\mathrm{Kpre}+\mathrm{CRc}$ (after hyperopic correction)

This method works very well, especially soon after refractive surgery. However, it becomes less accurate with time if the refraction changes either due to corneal changes, multiple refractive surgeries, or cataract development.

\section{Double-K Method to Correct the Estimated Lens Position}

In order to use certain third generation formulas such as SRK/T, Holladay, or Hoffer Q formulas, the second error of the ELP needs to be corrected. If not corrected, the greatest degree of error will be seen with the SRK/T and the least degree of error will be seen with the Hoffer Q formula. Again, the Haigis and Shammas-PL formulas do not need to correct the ELP.

Aramberri's double- $\mathrm{K}$ method is the best method to correct the ELP. In this method, the corrected post-LASIK $\mathrm{K}$ is used for the corneal power and the pre-LASIK $\mathrm{K}$ is used to calculate the ELP.

\section{Formulas Available}

There are many formulas that have been written to calculate the IOL power after refractive surgery. A more detailed explanation of the most reliable formulas has been 
Table 1 The most commonly used IOL formulas in post-refractive cases

\begin{tabular}{|c|c|c|c|}
\hline Method & $\begin{array}{l}\text { Pre-LASIK K } \\
\text { needed }\end{array}$ & $\begin{array}{l}\text { Change in MR } \\
\text { needed }\end{array}$ & Other information \\
\hline Clinical history* $\left[16^{\bullet}\right]$ & Yes & Yes & See explanation in text \\
\hline Feiz-Mannis [27] & Yes & Yes & $\begin{array}{l}\text { Use pre-LASIK K in IOL formula and add the change in MR divided } \\
\text { by } 0.7\end{array}$ \\
\hline Corneal bypass $[28,29]$ & Yes & Yes & $\begin{array}{l}\text { Use the pre-LASIK K in IOL formula and use the change in MR as the } \\
\text { target refraction }\end{array}$ \\
\hline Masket* $[11]$ & No & Yes & $\begin{array}{l}\text { Calculate IOL using the post-LASIK K (IOLpost }) \text { and adjust: } \\
\text { Adjusted IOL }=\text { IOLpost }+(0.326 \times \text { MR change })+0.101\end{array}$ \\
\hline Modified Masket* & No & Yes & $\begin{array}{l}\text { Masket formula is modified by Hill using: } \\
\text { Adjusted IOL }=\text { IOLpost }+(0.4385 \times \text { MR change })+0.0295\end{array}$ \\
\hline $\begin{array}{l}\text { Adjusted effective refractive } \\
\text { power (EffRP) }\end{array}$ & No & Yes & $\begin{array}{l}\text { Obtain the EffRP by the EyeSys corneal analysis system and adjust using: } \\
\text { Adjusted EffRP }=\text { EffRP }-(0.15 \times \text { MR change })-0.05\end{array}$ \\
\hline Adjusted atlas $0-3$ & No & Yes & $\begin{array}{l}\text { Obtain the mean corneal power from the Atlas topographer and adjust } \\
\text { using: } \\
\text { Adjust atlas = atlas }-0.2 \times \text { MR change }\end{array}$ \\
\hline Average ASCRS calculation & N/A & N/A & Averages the results of all formulas on the ASCRS website \\
\hline Shammas* [17] & No & No & $\begin{array}{l}\text { Use the Shammas-PL power formula with the adjust corneal power }(\mathrm{Kc}) \\
\text { where } \mathrm{Kc}=1.14 \mathrm{Kpost}-6.8\end{array}$ \\
\hline Haigis-L* $[18]$ & No & No & $\begin{array}{l}\text { Use the Haigis formula with an adjust corneal radius ( } \mathrm{r} \text { corr) using: } \\
\mathrm{R} \text { corr }=331.5 /(-5.1625 \times \mathrm{r} \text { meas }+82.2603-0.35)\end{array}$ \\
\hline Latkany flat K [10] & No & Yes & $\begin{array}{l}\text { IOL calculated using the flattest } \mathrm{K} \text { and } \mathrm{SRK} / \mathrm{T} \text { formula and adjusted using: } \\
\text { IOL adjusted }=\mathrm{Calc} \mathrm{IOL}-(0.47 \times \mathrm{MR} \text { change })+0.85\end{array}$ \\
\hline
\end{tabular}

* denotes the IOL formulas that are the most accurate according to multiple studies

included based on multiple review articles $\left[1^{\bullet}, 15^{\bullet}\right]$. See Table 1 for a more complete listing of formulas available.

\section{Clinical History Method}

The clinical history method is described in detail above. First, the correct post-LASIK corneal power is calculated. It is entered into a third generation IOL formula, such as SRK-T, Holladay 1, or the Hoffer Q formulas. The ELP is then determined using the double-K technique [16•].

\section{Masket and Modified Masket Formulas}

The Masket formula requires the knowledge of the surgically induced change in manifest refraction, MR, by the PRK or LASIK. The IOL power calculations are performed using the post-LASIK measured axial length and corneal power readings, and called IOLpost. For cases of myopic refractive surgery, Masket recommends the SRK/T formula whereas the Hoffer Q formula is recommended for hyperopic refractive surgery. The IOL power is then adjusted using the following equation [11]:

Adjusted IOL $=\mathrm{IOL}$ post $+(0.326 \times \Delta \mathrm{MR})+0.101$

Dr. Hill modified the Masket formula according to his cases using the following equation:
Adjusted $\mathrm{IOL}=\mathrm{IOL}$ post $+(0.4385 \times \Delta \mathrm{MR})+0.0295$

\section{Shammas-PL Formula}

The Shammas post-LASIK formula does not require historical data such as the pre-LASIK-K or change in manifest refraction. The Shammas post-LASIK formula is used with an adjusted corneal power, $\mathrm{Kc} . \mathrm{Kc}$ is found using the equation [17]:

$\mathrm{Kc}=1.14$ Kpost -6.8

Haigis-L Formula

The Haigis-L formula also does not need historical data such as the post-LASIK K or change in manifest refraction. The Haigis formula is used with an adjusted corneal radius of curvature, $r$ corr. It is derived from the radius of curvature measured by the IOL master, $r$ meas, and uses the equation [18]:

$\mathrm{r}$ corr $=331.5 /(-5.1625 \times \mathrm{r}$ meas $+82.2603-0.35)$

\section{Post-Hyperopic Refractive Surgery}

Corneal Changes After Hyperopic Refractive Surgery

IOL calculations after hyperopic refractive surgery can be approached in a similar manner to that of myopic refractive 
surgery. The corneal changes do differ amongst the two and the degree of error becomes less when dealing with the posthyperopic LASIK or PRK patient. One reason is that the ablation zone is out of the central cornea as it works to steepen the cornea. Because of this, there is less error in keratometry if it measures the corneal power within the central $3 \mathrm{~mm}$. Also, the amount of correction in a hyperopic LASIK or PRK tends to be less, usually between 1 and 3 diopters.

\section{Correcting the Errors}

Regardless of these differences, the corneal power tends to be under-estimated and the ELP value is over-estimated. The same formulas described in the above sections can be used to correct these errors. When using the double-K method, two considerations can be made. Because the central corneal is not significantly altered in these cases, the average corneal power rather than Sim-K values may yield more consistent results, and one can use that measurement when adjusting the corneal power to the amount of refractive change $[19,20]$. Also, one can consider using the Hoffer Q formula since it performs better in shorter, hyperopic eyes [19]. If no prior data are known, the Haigis$\mathrm{L}$ and the Shammas-PHL formulas use different algorithms to measure IOL power after hyperopic LASIK or PRK.

\section{Post-Radial Karatectomy (RK)}

\section{Corneal Changes After RK}

Radial keratotomy, RK, aims to correct myopia via deep radial cuts into the anterior cornea, which flattens both the anterior and the posterior corneal curvature, affecting only a small central optical zone [21]. The anterior to posterior corneal curvature ratio stays intact, but the overall corneal power changes. Standard keratometry tends to overestimate the corneal power. The ELP estimation used in many third generation formulas will be altered because the overall corneal curvature has changed [16•]. These errors combined with post-cataract extraction corneal edema results in hyperopic and variable outcomes [22, 23].

\section{Correcting the Errors}

RK is no longer the favored refractive surgery; however, we continue to encounter these patients for cataract surgery. A large hyperopic outcome is not acceptable to these patients. One method, advised by Chen et al. [23], simply aims for a -1.50 Diopter post-refractive outcome. This method works fairly and will often get one close to an acceptable result.

To yield the most accurate keratometric results, data from only the central $3 \mathrm{~mm}$ should be taken $[24,25]$.
Manual and auto-keratometry are difficult in these cases, especially if the cuts are more numerous and impinge on the central $3 \mathrm{~mm}$. Corneal topography and Scheimpflug photography are helpful in these cases. Awwad et al. [26] used the average of all the topographic data within the central $3 \mathrm{~mm}$ area of the cornea and yielded predictable and accurate outcomes.

To limit error from the estimation of ELP in the IOL formulas, one can use the Haigis formula because it does not depend on the corneal power for ELP estimation. Alternatively, if the other formulas are to be used, Aremberri's double-K method, as described in the myopic refractive cases, should be used [16•]. The corneal power measured post-RK is used in the SRK/T formula for the K, but is replaced with the corneal power pre-RK in the part of the equation that estimates the ELP [16•]. If there is no known information of the pre-RK corneal power, one can use that of the general population, 43.86 [26].

In general, one can use the Haigis formula inputting the steepest corneal power taken from the keratometer. When entered into the formula, the IOL power is calculated without any further adjustment.

\section{Additional Resources}

The ASCRS Website www.ascrs.org

The ASCRS website is an IOL calculator for prior myopic or hyperopic LASIK/PRK or RK. The program will then give an average IOL power along with the minimal and maximal calculated powers (Table 1).

\section{The Hoffer/Savini Tool}

The Hoffer/Savini Tool uses an Excel sheet downloadable from the Eye Lab-Hoffer website; one can use all the modern formulas in post-LASIK/PRK eyes.

\section{The Holladay IOL Consultant Software}

The Holladay IOL Consultant program calculates the IOL power with the Holladay 2 formula with its enhanced algorithm, and compares the results to the Holladay 1, Hoffer Q, and SRK/T formulas. It will correct the formulas from the refractive data and topography in post-refractive cases.

\section{Conclusion}

The corneal changes in refractive surgery have posed many challenges when calculating the intraocular lens. By 
understanding how these changes affect the formulas used, one can make better decisions in IOL choice.

The clinical history method, the Feiz-Mannis, and the corneal bypass methods are the most accurate, especially soon after refractive surgery. However, they can be less reliable over time if the patient experiences corneal changes, multiple refractive surgeries, or cataract progression. The Masket and the modified Masket formulas have been reported to be quite accurate if the change in manifest refraction has remained stable. The Haigis-L and the Shammas-PL formulas can be used in every case, especially if the refractive changes produced by the LASIK/ PRK are not known.

In general, it is best to input the most data one can obtain into one of the above-mentioned tools. This will allow you to compare the calculations of multiple formulas. In our experience, and depending on the situation, we find that one can often average the different calculated IOL power values aiming slightly myopic. Consider discarding any outliers. In all cases, having a discussion with each patient and having a section in the informed consent dedicated to post-refractive surgery will allow for a happy and informed patient.

Disclosure No potential conflicts of interest relevant to this article were reported.

\section{References}

Papers of particular interest, published recently, have been highlighted as:

- Of importance

1. - McCarthy M, et al. Intraocular lens power calculations after myopic laser refractive surgery: a comparison of methods in 173 eyes. Ophthalmology. 2011;118(5):940-4. This retrospective case series evaluates the accuracy of various formulas in a relatively large study.

2. Seitz B, et al. Underestimation of intraocular lens power for cataract surgery after myopic photorefractive keratectomy. Ophthalmology. 1999;106(4):693-702.

3. Hugger $\mathrm{P}$, et al. Comparison of changes in manifest refraction and corneal power after photorefractive keratectomy. Am J Ophthalmol. 2000;129(1):68-75.

4. Gobbi PG, Carones F, Brancato R. Keratometric index, videokeratography, and refractive surgery. J Cataract Refract Surg. 1998;24(2):202-11.

5. Bastian G, et al. Impact of UFT on tumoral TS and DPD levels in colorectal cancer. Oncology (Williston Park). 2000;14(10 Suppl 9):35-7.

6. Holladay JT, et al. A three-part system for refining intraocular lens power calculations. J Cataract Refract Surg. 1988;14(1):17-24.

7. Retzlaff JA, Sanders DR, Kraff MC. Development of the SRK/T intraocular lens implant power calculation formula. J Cataract Refract Surg. 1990;16(3):333-40.
8. Wilson SE, et al. Changes in corneal topography after excimer laser photorefractive keratectomy for myopia. Ophthalmology. 1991;98(9):1338-47.

9. Seitz B, Langenbucher A. Intraocular lens calculations status after corneal refractive surgery. Curr Opin Ophthalmol. 2000;11(1):35-46.

10. Latkany RA, et al. Intraocular lens calculations after refractive surgery. J Cataract Refract Surg. 2005;31(3):562-70.

11. Masket S, Masket SE. Simple regression formula for intraocular lens power adjustment in eyes requiring cataract surgery after excimer laser photoablation. J Cataract Refract Surg. 2006;32(3):430-4.

12. Hamilton DR, Hardten DR. Cataract surgery in patients with prior refractive surgery. Curr Opin Ophthalmol. 2003;14(1):44-53.

13. Chan CC, Hodge C, Lawless M. Calculation of intraocular lens power after corneal refractive surgery. Clin Exp Ophthalmol. 2006;34(7):640-4.

14. Shammas HJ, et al. Correcting the corneal power measurements for intraocular lens power calculations after myopic laser in situ keratomileusis. Am J Ophthalmol. 2003;136(3):426-32.

15. - Savini G, et al. Intraocular lens power calculation after myopic excimer laser surgery: clinical comparison of published methods. J Cataract Refract Surg. 2010;36(9):1455-65. This is a prospective case series that compares the accuracy of various formulas.

16. - Aramberri J. Intraocular lens power calculation after corneal refractive surgery: double-K method. J Cataract Refract Surg. 2003;29(11):2063-8. This article describes and evaluates the double-K method for IOL calculations post refractive surgery.

17. Shammas HJ, Shammas MC. No-history method of intraocular lens power calculation for cataract surgery after myopic laser in situ keratomileusis. J Cataract Refract Surg. 2007;33(1):31-6.

18. Haigis W. Intraocular lens calculation after refractive surgery for myopia: Haigis-L formula. J Cataract Refract Surg. 2008;34(10): 1658-63.

19. Awwad ST, et al. Corneal refractive power estimation and intraocular lens calculation after hyperopic LASIK. Ophthalmology. 2009;116(3):393-400.

20. Wang L, Jackson DW, Koch DD. Methods of estimating corneal refractive power after hyperopic laser in situ keratomileusis. J Cataract Refract Surg. 2002;28(6):954-61.

21. Waring GO III, Lynn MJ, McDonnell PJ. Results of the prospective evaluation of radial keratotomy (PERK) study 10 years after surgery. Arch Ophthalmol. 1994;112(10):1298-308.

22. Bardocci A, Lofoco G. Corneal topography and postoperative refraction after cataract phacoemulsification following radial keratotomy. Ophthalmic Surg Lasers. 1999;30(2):155-9.

23. Chen $\mathrm{L}$, et al. Analysis of intraocular lens power calculation in postradial keratotomy eyes. J Cataract Refract Surg. 2003;29(1):65-70.

24. Maeda N, et al. Disparity between keratometry-style readings and corneal power within the pupil after refractive surgery for myopia. Cornea. 1997;16(5):517-24.

25. Packer M, et al. Intraocular lens power calculation after incisional and thermal keratorefractive surgery. J Cataract Refract Surg. 2004;30(7):1430-4.

26. Awwad ST, et al. Intraocular lens power calculation after radial keratotomy: estimating the refractive corneal power. J Cataract Refract Surg. 2007;33(6):1045-50.

27. Feiz V, et al. Intraocular lens power calculation after laser in situ keratomileusis for myopia and hyperopia: a standardized approach. Cornea. 2001;20(8):792-7.

28. Ladas JG, Stark WJ. Calculating IOL power after refractive surgery. J Cataract Refract Surg. 2004;30(12):2458. author reply 2458-9.

29. Walter KA, et al. Accurate intraocular lens power calculation after myopic laser in situ keratomileusis, bypassing corneal power. J Cataract Refract Surg. 2006;32(3):425-9. 